\title{
Normal Range Comparison Code
}

National Cancer Institute

\section{Source}

National Cancer Institute. Normal Range Comparison Code. NCI Thesaurus. Code C93603.

A coded value specifying the relationship of a value to a normal range or reference range of values. 